\title{
Relationships between patient- and session-related variables and outcomes of psychiatric genetic counseling
}

\author{
Sarah Gerrard ${ }^{1} \cdot$ Angela Inglis 2,3 Emily Morris ${ }^{2,3} \cdot$ Jehannine Austin $\mathbb{D}^{2,3}$
}

Received: 29 October 2019 / Revised: 9 January 2020 / Accepted: 22 January 2020 / Published online: 17 February 2020

(c) The Author(s), under exclusive licence to European Society of Human Genetics 2020

\begin{abstract}
Little data currently exist regarding whether and how different characteristics of a patient and session influence outcomes of genetic counseling (GC). We conducted an exploratory retrospective chart review of data from a specialist psychiatric GC clinic (where patients complete the Genetic Counseling Outcome Scale (GCOS) as part of routine care before and after GC). We used ANOVA and linear regression to analyze GCOS change scores in relation to twelve patient/session-related variables. Three hundred and seven charts were included in analyses. Overall, GCOS scores increased significantly after GC, with large effect size $(p<0.0005, d=1.10)$, and significant increases in all GCOS subdomains except adaptation. Significant associations with GCOS change score were identified for three variables: mode of delivery of GC (in-person/telephone/ telehealth, $p=0.048, \eta^{2}=0.020$ ), primary indication for the appointment (understanding recurrence risk versus other primary indications, $\left.p=0.001, \eta^{2}=0.037\right)$, and baseline GCOS score $(p<0.000, R=0.353)$. Our data showing that those with low baseline GCOS scores benefit most from GC could be used to explore the possibility of triaging those referred for GC based on this variable, and/or to identify individuals to refer to GC.
\end{abstract}

\section{Introduction}

The genetic counseling $(\mathrm{GC})$ profession has recognized the importance of identifying and measuring patient outcomes $[1,2]$. GC outcomes research has historically focused on cancer GC [3-6] and most studies have used simple prepost study design and the assessment of knowledge/satisfaction-based outcomes [7]. There has been comparatively less research addressing psychological outcomes of GC, especially outside of the cancer context, and very little research exploring the effects of patient- or session-related

Supplementary information The online version of this article (https:// doi.org/10.1038/s41431-020-0592-1) contains supplementary material, which is available to authorized users.

Jehannine Austin

jehannine.austin@ubc.ca

1 Joan H. Marks Graduate Program in Human Genetics, Sarah Lawrence College, Bronxville, NY, USA

2 Department of Psychiatry, University of British Columbia, Vancouver, Canada

3 Department of Medical Genetics, University of British Columbia, Vancouver, Canada variables on these outcomes. Studies have explored patient outcomes of GC in relation to: patient age and education level [4], sex and referral indication [8], mode of service delivery $[9,10]$, method of family history collection [11], provision of chance for illness recurrence [12], and physical counseling environment [13], and have revealed few relationships between patient outcomes and the studied variables that are both statistically and clinically significant. However, important knowledge gaps remain. For example, in the psychiatric context, though GC is associated with important benefits to patients, including marked increases in empowerment [11, 12, 14-19], no studies have explored the relationships between empowerment and patient/sessionrelated variables such as sex, ethnicity, diagnosis, mode of referral or mode of GC. Furthermore, though studies have explored how different domains of the empowerment construct are more substantially impacted by the provision of GC in other areas $[8,20]$ this has yet to be explored in the context of psychiatric GC.

Given that understanding factors that influence patient outcomes of GC at a more nuanced level may allow for prioritizing patients who might benefit most, or for adjustment of service delivery strategies to promote the best possible outcomes for difference types of patients, we set out to analyze - in an exploratory manner-the change in 
empowerment (as measured by the Genetic Counseling Outcome Scale (GCOS) [21], from pre- to post-psychiatric $\mathrm{GC}$ in relation to twelve patient/session variables. In addition, we sought to examine-again in an exploratory manner-the effect of psychiatric GC on individual GCOS items and domains.

\section{Materials and methods}

We conducted a retrospective chart review using data collected at a specialist psychiatric GC clinic in Vancouver, BC. This study was approved by the BC Children and Women's Research Ethics Board (H15-02632).

\section{Clinical context}

Broadly, GC is designed to help people to "understand and adapt to the medical psychological and familial implications of genetic contributions to disease" [22]. In the psychiatric context more specifically, GC aims to help people understand how genes and environment contribute together to the development of illness, how to protect their mental health, and to address the guilt, fear, blame, shame, and stigma that are often attached to people's explanations for causes of these conditions. The content and structure of the psychiatric GC appointment are generally consistent between sessions (i.e., regardless of indication of referral, etiology of mental illness and strategies for protecting mental health are discussed in a personalized manner, and emotional issues related to explanations for cause of illness are explored), with specific numeric estimates of risk for recurrence provided according to patient wishes [12]. Details of the process and structure of the session, including common core elements (in the form of a manual) have been described in detail elsewhere [23] In qualitative explorations, patients have described their experience with psychiatric GC as "an empowering encounter" [24], and quantitative studies show marked increases in patient empowerment [11, 12, 14-19] after psychiatric GC.

GC appointments are covered by the publicly funded healthcare system for all residents of British Columbia and are provided by two board certified genetic counselors. The clinic uses the GCOS as a clinical assessment tool; it is typically completed by all English-speaking patients at the beginning of their GC appointment (T1), and again at a standard follow-up telephone appointment (T2) approximately 1-2 months post GC. This service is available to anyone with a personal and/or family history of a psychiatric disorder, and all clinical data (including demographic information and GCOS scores from $\mathrm{T} 1$ and $\mathrm{T} 2$ ) are collected and managed using REDCap (Research Electronic Data Capture) tools hosted at BC Children's and Women's Hospital [25]. REDCap is a secure, web-based application designed to support data capture for research purposes, providing (1) an intuitive interface for validated data entry; (2) audit trails for tracking data manipulation and export procedures; (3) automated export procedures for seamless data downloads to common statistical packages; and (4) procedures for importing data from external sources.

\section{Inclusion criteria}

We extracted data from charts of index patients (family members were excluded) who attended their first appointment between February 1, 2012 and January 31, 2017, and who had completed the GCOS (defined as $\leq 5$ missing items) at both timepoints.

\section{Genetic Counseling Outcome Scale (GCOS)}

The GCOS is a validated, clinical genetics-specific patientreported outcome measure that measures empowerment [21]. All 24 items are rated on a 7-point Likert scale $(1=$ strongly disagree, $7=$ strongly agree). Scores range from 24 to 168 with higher scores indicating higher levels of empowerment. The scale comprises of seven subdomains, or putative subscales: hope, powerlessness, emotional regulation, adaptation, referral clarity, support, and family impact [20].

\section{Patient/session variables}

We assessed change in GCOS scores in relation to 12 variables about which data were available, specifically: age, sex, ethnicity, mode of referral (self or healthcare provider), mode of GC (in-person, telephone or telehealth), primary indication for referral, type of appointment (family or individual), GC student involvement (yes or no), presence of observers (e.g., visiting trainee/physicians)(yes or no), history of mental illness (personal or family), diagnosis, and baseline (T1) GCOS score.

\section{Analyses}

Descriptive statistics were applied to the demographic data, and GCOS total scores at $\mathrm{T} 1$ and $\mathrm{T} 2$ were calculated according to instrument-specific instructions. We described the mean pre- and post-GC scores, and mean change scores, for each GCOS item using data from the entire cohort. In addition, we calculated the mean change score for each of the seven GCOS subdomains and conducted a paired sample $t$ test to examine change in GCOS scores for each subdomain. We calculated Cronbach's alpha for the scale as a whole, and for each of the subdomains at both timepoints.

Data were examined for continuity, independence of observations, homogeneity of variance and normality before conducting one-way between-group analyses of variance 
(ANOVAs) for all of the variables (except T1 GCOS score), using mean GCOS change scores (T2-T1), with Tukey's HSD post hoc tests where applicable. To assess the effect of baseline GCOS score on change in GCOS score, we used a linear regression. Given that this was an exploratory, hypothesis generating study, we used a significance threshold of $p<0.05$ for all tests. To provide context for the analyses, we conducted a paired sample $t$ test to compare the change in GCOS scores from T1 to T2 for the cohort as a whole. We excluded any group of $n=1$ from analyses. Changes in GCOS scores were considered in light of a threshold change score of 10.3, that was determined in a previous study to correlate with the minimum clinically important difference (MCID) [26]. All analyses were performed using IBM SPSS Statistics 24 (IBM Corp., Armonk, N.Y., USA).

\section{Results}

There were 307 charts in the clinical database that met the inclusion criteria. Demographic data for the cohort of patients included in the analyses are shown in Table 1.

Cronbach's alpha for all 24 items of the GCOS was 0.827 at $\mathrm{T} 1$, and 0.845 at $\mathrm{T} 2$, thus showing good reliability. Overall, GCOS scores increased from T1 to T2 (T1: $M=$ 111.09, $\mathrm{SD}=17.68, \mathrm{~T} 2: M=127.17, \quad \mathrm{SD}=18.20, p<$ $0.0005, d=1.10$ ), with mean increases in score being greater than the MCID [22]. Assumptions for continuity, independence of observations, homogeneity of variance and normality were met. At the individual level, GCOS scores increased for $86 \%$ of patients (see Table 1).

For each item of the GCOS, the average T1 and T2 (preand post-GC, respectively) scale scores, and average change score is described in Supplementary Table 1. For each subdomain of the GCOS, Cronbach's alpha, average preand post-GC scores, and average change scores are described in Table 2. The subdomains of the GCOS where GC had the greatest effect were powerlessness and emotional regulation (Table 2).

\section{Change in GCOS scores in relation to patient/session variables}

All data regarding patient- and session-related variables are provided in Table 3. There was no significant difference in GCOS change scores (T2-T1) according to age $(F(1,305)=$ $3.357, p=0.068)$, sex $(F(1,304)=2.158, p=0.143)$, ethnicity $(F(4,290=0.981), p=0.418)$, mode of referral $(F(1$, $305)=1.266, p=0.261)$, type of appointment $(F(1,305)=$ $0.326, p=0.568)$, GC student involvement $(F(1,299)=$ $0.036, p=0.851)$, presence of observers $(F(1,167=0.061$, $p=0.805)$, or personal versus family history of mental illness $(F(1,305)=1.233, p=0.268)$.
Table 1 Demographic information.

\begin{tabular}{ll}
\hline & All patients $N=307$ \\
\hline Age [mean (SD)] & $41.13(12.09)$ \\
Sex $[n(\%)]$ & $50(16.3)$ \\
Male & $256(83.4)$ \\
Female & $1(0.3)$ \\
Other & \\
Ethnicity $[n(\%)]$ & $208(67.8)$ \\
European & $46(15.0)$ \\
Asian & $1(0.3)$ \\
Aboriginal & $3(1)$ \\
African & $34(11.1)$ \\
Mixed & $3(1)$ \\
Other & $12(3.9)$ \\
Unknown & $111.09(17.68)^{\mathrm{a}}$ \\
GCOS T1 scores [mean (SD)] & $127.17(18.20)^{\mathrm{a}}$ \\
GCOS T2 scores [mean (SD)] & $16.08(14.63)$ \\
Change scores (T2-T1) [mean (SD)] & $6(2)$ \\
Change score category $[n(\%)$, mean change scores (SD)] \\
Any increase & $265(86.3), 19.68(12.01)$ \\
Increase $\geq$ MCID & $209(78.9), 23.5(10.6)$ \\
Increase $<$ MCID & $56(21.1), 5.44(2.84)$ \\
Decrease & $36(11.7),-7.74(7.49)$ \\
No change & 6 \\
\hline
\end{tabular}

${ }^{\mathrm{a}} p=<0.0005$, Cohen's $d=1.10$.

${ }^{\mathrm{b}}$ Minimum clinically important difference $=10.3$.

A significant relationship was found between GCOS change scores and mode of $\mathrm{GC}(F(2,304)=3.067, p=$ 0.048). The effect size was small $\left(\eta^{2}=0.020\right)$. Though changes in GCOS scores were numerically greater for the in-person counseling group compared with the telephone and telehealth groups (by 4.62 and 6.31 points, respectively), the differences between groups were not statistically significant according to Tukey's post hoc test $(p=0.111$ and $p=0.234$, respectively).

GCOS change scores were greater for patients who stated that recurrence risk was a primary indication for referral, compared with the individuals who did not indicate this referral indication $(F(1,305)=11.624, p=0.001)$. The effect size was small to medium $\left(\eta^{2}=0.037\right)$. There were no significant differences in GCOS change scores when we compared the other primary indications individually (see Table 3 ).

Mean increases in GCOS scores were greater than MCID for all categorical variables, with the exception of those with a diagnosis of schizophrenia or schizoaffective disorder, but the number of individuals in these groups were too small to draw meaningful conclusions (see Table 3).

GCOS change scores were significantly related to baseline GCOS scores $\left(F(1,304)=43.8, p<0.000, R^{2}=0.125\right)$, with a moderate effect size $(R=0.353)$ : specifically, we found a 
Table 2 Subdomains of GCOS: T1 and T2, change (T2-T1), and Cronbach's alpha.

\begin{tabular}{|c|c|c|c|c|c|c|c|}
\hline & \multicolumn{2}{|l|}{$\mathrm{T} 1$} & \multicolumn{2}{|l|}{$\mathrm{T} 2$} & \multirow{2}{*}{$\begin{array}{l}\text { Change (SD) } \\
(\mathrm{T} 2-\mathrm{T} 1)\end{array}$} & \multirow[t]{2}{*}{$p$} & \multirow[t]{2}{*}{ Cohen's $d$} \\
\hline & $\begin{array}{l}\text { GCOS } \\
\text { mean }(\mathrm{SD})\end{array}$ & Cronbach's alpha & $\begin{array}{l}\text { GCOS } \\
\text { mean }(\mathrm{SD})\end{array}$ & Cronbach's alpha & & & \\
\hline Hope & $5.32(0.37)$ & 0.748 & $5.76(0.30)$ & 0.775 & $0.44(0.12)$ & 0.005 & 1.19 \\
\hline Support & $4.94(0.59)$ & 0.626 & $5.63(0.50)$ & 0.642 & $0.69(0.14)$ & $<0.0001$ & 1.17 \\
\hline $\begin{array}{l}\text { Emotional } \\
\text { regulation }\end{array}$ & $3.29(0.27)$ & 0.575 & $3.96(0.46)$ & 0.667 & $0.67(0.25)$ & 0.045 & 2.48 \\
\hline Family impact & $4.02(0.75)$ & 0.482 & $5.15(0.66)$ & 0.520 & $1.13(0.20)$ & 0.010 & 1.5 \\
\hline Powerlessness & $4.40(0.37)$ & 0.548 & $5.39(0.05)$ & 0.613 & $0.99(0.38)$ & 0.045 & 2.68 \\
\hline Referral clarity & $5.72(0.54)$ & 0.561 & $6.21(0.51)$ & 0.510 & $0.49(0.05)$ & 0.003 & 0.91 \\
\hline Adaptation & $3.95(0.98)$ & 0.561 & $4.36(1.05)$ & 0.573 & $0.41(0.23)$ & 0.094 & 0.42 \\
\hline
\end{tabular}

linear relationship between the two (See Fig. 1), those with lower baseline GCOS sores had greater increases in GCOS scores after GC. This model shows that a baseline GCOS score of $\leq 131$ predicts meeting or surpassing the MCID GCOS change score of 10.3 [26]. Those with baseline GCOS scores higher than 131 are predicted to have increases in GCOS scores smaller than the MCID threshold. The demographic characteristics of those above and below this threshold baseline (T1) GCOS score are shown in Table 4; those who indicated a desire to discuss protective factors had higher baseline GCOS scores, and older individuals and those with a family history of mental illness rather than a personal history had lower baseline GCOS scores.

The characteristics of those with GCOS change scores above and below the MCID threshold are shown in Supplementary Table 2 .

When we examined individuals who only had one diagnosis, there was no significant difference in GCOS change scores according to diagnosis $(p=0.283)$, however the effect size was medium $\left(\eta^{2}=0.056\right)$. Additional data regarding pre-, post- and change scores by diagnosis for individuals with multiple diagnoses are shown in Supplementary Table 3.

\section{Discussion}

This study represents the first examination of how these specific patient- and session-related variables influence patient outcomes of psychiatric GC, and the first examination of the impact of psychiatric GC on individual items and subdomains of the GCOS. Overall, our data show significant increases in levels of empowerment from before to after GC. There were also significant increases with large effect sizes in all of the subdomains of empowerment except adaptation, where the effect was moderate and nonsignificant at a threshold of 0.05 . In this regard, our study aligns with the findings of Ison et al., who also found significant improvement in post-GC scores in six of the seven subdomains, with adaptation being the subdomain that was not significant [8]. We found that the subdomains of empowerment on which psychiatric GC had the largest effect were powerlessness and emotional regulation. Though we cannot directly compare data with the findings of Costal-Tirado et al. (as effect sizes were not reported) emotional regulation was the one subdomain of empowerment in their study in which significant improvements were not observed from pre- to post-GC. This raises interesting questions about differences in GC outcomes between different patient populations, and/or different practice models of GC that are worthy of further exploration.

With regard to the influence of patient- and sessionrelated variables on GC outcomes, we found that several of those we studied had no significant relationship with change in empowerment associated with receiving psychiatric GC. These included: age, sex, ethnicity, self-referral versus referral from a healthcare provider, individual versus family appointment, the involvement of students or observers, and personal versus family history of mental illness.

Though as far as we are aware, there is no previous data with which to compare it, our finding that the presence of observers or students did not influence GC outcomes will be reassuring for trainees, who may worry that they negatively impact the quality of a patient's care. Similarly, while anecdotally/from clinical experience, family appointments can be more challenging for the genetic counselor to manage, it may be assuring to providers to see that this complexity is not accompanied by a negative influence on patient outcomes.

For some of the other variables studied, data are available with which to compare our findings. For example, in other GC contexts (non-psychiatric) age has been found to influence outcomes of GC; specifically, older participants had smaller increases in knowledge after cancer GC [4]. However, one of the key differences is the outcome variable being assessed; knowledge in the cancer study, and empowerment in the data reported here: variables that affect 
Table 3 GCOS change scores for categorical variables (one-way between groups ANOVA).

\begin{tabular}{|c|c|c|c|c|c|c|}
\hline & $N$ & $\mathrm{~T} 1$ mean $(\mathrm{SD})$ & $\mathrm{T} 2$ mean $(\mathrm{SD})$ & $\begin{array}{l}\text { Change (SD) } \\
\text { (T2-T1) }\end{array}$ & $\begin{array}{l}\text { ANOVA } \\
p \text { value }\end{array}$ & $\eta^{2}$ \\
\hline \multicolumn{7}{|l|}{ Age } \\
\hline $13-40$ & 160 & 113.12 (17.27) & $130.66(16.78)$ & $17.54(14.65)$ & \multirow[t]{2}{*}{0.068} & \multirow[t]{2}{*}{0.011} \\
\hline $41-77$ & 147 & $108.88(17.90)$ & 123.37 (18.97) & $14.49(14.49)$ & & \\
\hline \multicolumn{7}{|l|}{ Sex } \\
\hline Male & 50 & $111.65(17.15)$ & $125.05(19.50)$ & $13.40(17.26)$ & \multirow[t]{2}{*}{0.143} & \multirow[t]{2}{*}{0.007} \\
\hline Female & 256 & $110.93(17.82)$ & $127.63(17.97)$ & $16.70(13.97)$ & & \\
\hline \multicolumn{7}{|l|}{ Ethnicity } \\
\hline European & 208 & $111.38(17.42)$ & $128.30(17.69)$ & $16.92(14.60)$ & \multirow[t]{5}{*}{0.418} & \multirow[t]{5}{*}{0.013} \\
\hline Asian & 46 & $113.48(15.73)$ & $126.93(17.83)$ & $13.45(14.05)$ & & \\
\hline African & 3 & 95.29 (17.28) & $111.29(29.65)$ & $16.00(12.62)$ & & \\
\hline Mixed & 34 & $107.22(22.22)$ & $124.46(17.18)$ & $17.23(13.56)$ & & \\
\hline Other & 4 & 109.17 (21.06) & $116.37(36.05)$ & $7.20(16.55)$ & & \\
\hline \multicolumn{7}{|l|}{ Mode of referral } \\
\hline Self-referral & 114 & $109.62(16.38)$ & $124.48(18.12)$ & $14.86(15.61)$ & \multirow[t]{2}{*}{0.261} & \multirow[t]{2}{*}{0.004} \\
\hline Healthcare provider & 193 & $111.96(18.38)$ & $128.76(18.11)$ & $16.80(14.01)$ & & \\
\hline \multicolumn{7}{|l|}{ Mode of GC } \\
\hline Telephone & 48 & 111.08 (19.54) & $123.57(18.47)$ & $12.49(13.35)$ & \multirow[t]{3}{*}{0.048} & \multirow[t]{3}{*}{0.020} \\
\hline In-person & 244 & $110.89(17.22)$ & $128.00(18.13)$ & $17.11(14.84)$ & & \\
\hline Telehealth & 15 & 114.37 (19.64) & $125.17(18.15)$ & $10.80(12.90)$ & & \\
\hline \multicolumn{7}{|l|}{ Primary indication } \\
\hline Recurrence risk & 147 & $110.87(17.56)$ & $129.87(16.04)$ & $19.00(13.83)$ & 0.001 & 0.037 \\
\hline Understanding causes & 189 & $110.47(17.80)$ & $127.26(18.58)$ & $16.79(14.72)$ & 0.285 & 0.004 \\
\hline Protective factors & 81 & $114.76(16.98)$ & $129.69(18.11)$ & $14.93(12.52)$ & 0.411 & 0.002 \\
\hline Had genetic testing & 4 & $93.39(18.03)$ & $110.00(23.76)$ & $16.61(17.17)$ & 0.942 & 0.000 \\
\hline Pregnancy related & 17 & $121.74(15.41)$ & $134.50(15.50)$ & $12.76(9.37)$ & 0.337 & 0.003 \\
\hline Other & 6 & $98.00(10.55)$ & $122.50(13.03)$ & $24.50(10.03)$ & 0.155 & 0.007 \\
\hline Unsure & 20 & $117.19(13.98)$ & $127.49(21.56)$ & $10.30(17.84)$ & 0.067 & 0.011 \\
\hline \multicolumn{7}{|l|}{ Type of appointment } \\
\hline Family & 89 & $110.72(16.16)$ & $127.54(16.63)$ & $16.83(15.60)$ & \multirow[t]{2}{*}{0.568} & \multirow[t]{2}{*}{0.001} \\
\hline Individual & 218 & $111.24(18.29)$ & $127.02(18.84)$ & $15.78(14.24)$ & & \\
\hline \multicolumn{7}{|l|}{ GC student involvement } \\
\hline Yes & 72 & $112.45(17.23)$ & $128.51(20.62)$ & $16.06(13.45)$ & \multirow[t]{2}{*}{0.851} & \multirow[t]{2}{*}{0.000} \\
\hline No & 229 & $110.59(17.70)$ & $127.03(17.23)$ & $16.44(14.99)$ & & \\
\hline Presence of observer & & & & & & \\
\hline Yes & 38 & $116.31(15.20)$ & $132.45(17.18)$ & $16.13(11.82)$ & 0.805 & 0.000 \\
\hline No & 131 & $111.51(17.92)$ & $127.04(18.11)$ & $15.53(13.75)$ & & \\
\hline History of mental illness & & & & & & \\
\hline Personal history & 259 & $111.16(18.10)$ & $127.64(18.35)$ & $16.48(14.61)$ & 0.268 & 0.000 \\
\hline Family history only & 48 & $110.73(18.10)$ & $124.66(18.35)$ & $13.93(14.72)$ & & \\
\hline Personal history & & & & & & \\
\hline Schizophrenia & 5 & $114.40(19.58)$ & $119.96(14.83)$ & $5.56(7.36)$ & 0.283 & 0.056 \\
\hline Bipolar disorder & 30 & $110.97(20.79)$ & $129.95(19.53)$ & $18.98(19.01)$ & & \\
\hline Schizoaffective & 4 & $116.91(17.60)$ & $121.76(28.58)$ & $4.85(20.52)$ & & \\
\hline Anxiety & 17 & $118.06(12.49)$ & $134.10(10.61)$ & $16.04(13.51)$ & & \\
\hline Depression & 53 & $111.44(17.38)$ & $129.02(18.37)$ & $17.59(13.04)$ & & \\
\hline Other & 3 & $110.67(4.04)$ & $130.67(6.11)$ & $20.00(2.65)$ & & \\
\hline
\end{tabular}


Fig. 1 Relationship between baseline GCOS score and change in GCOS after genetic counseling. The model shows a linear relationship between baseline GCOS score and change in GCOS after genetic counseling (predicted change in GCOS $=47.068+-0.281$ baseline GCOS). The dotted lines indicate the MCID threshold (10.3) on the $y$-axis, and the threshold baseline its corresponding baseline GCOS (131).

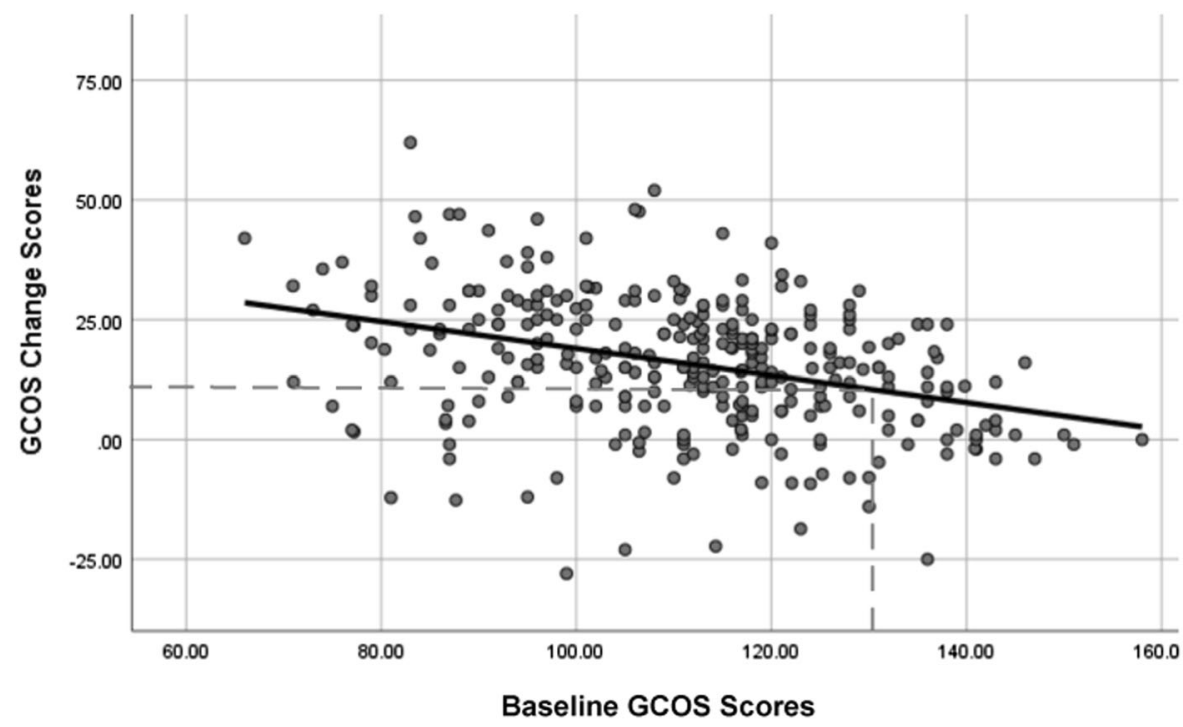

knowledge-based outcomes will not necessarily influence emotional or psychological outcomes.

Another area in which data exist with which to compare our findings related to self-, versus healthcare provider referrals. Some previous studies have suggested that individuals who self-refer are more likely to change health behaviors in response to the information they receive, compared with those who are referred by a healthcare provider in clinical genetics and healthcare services $[27,28]$. Relatedly, it has been suggested that those who self-refer may have higher levels of anxiety, or other psychosocial variables that may play a role in their response to treatment $[29,30]$. However, our data showed no difference in GCOS scores between those who self-referred and those who were referred by a healthcare provider.

A third area in which some data exist with which to compare our findings is mode of delivery; i.e., in-person versus telephone/telehealth GC. In the current study, though the initial ANOVA suggested a marginally statistically significant difference in change in empowerment according to mode of delivery (with those who received GC in person having larger gains in empowerment) it did not survive Tukeys post hoc testing, perhaps due to discrepancies between group sizes - only 48 patients received telephone GC, and 15 patients were seen through telehealth, compared with 244 patients who attended the GC appointments inperson. Though we found no previous research that has explored the impact of mode of GC on empowerment specifically, patient satisfaction has been compared between those receiving cancer GC by telegenetics and those receiving in-person service. The study revealed no differences in this measure between groups, but identified the need for further randomized trials to compare longer term psychosocial and behavioral outcomes [9]. It is possible that while acceptability of the two modes of delivery is comparable (as assessed by satisfaction), the effectiveness may differ (e.g., a possible small effect on empowerment). Adding some degree of credence to this possibility, one study found telephone counseling to be noninferior to inperson counseling for a variety of measures including both satisfaction, and distress and decisional conflict, but rate of testing uptake differed between groups [10].

We identified a significantly greater increase in levels of empowerment for patients for whom understanding recurrence risk was a primary indication for referral, compared with patients with other primary indications $(p=0.001$, $\eta^{2}=0.037$ ). Data from Borle et al. may provide some insight into this finding: specifically, in this study $27 \%$ of individuals who initially indicated that their primary motivation for $\mathrm{GC}$ was to receive recurrence risk estimates changed their minds after discussing etiology and protective factors. This subset of patients had significantly greater increases in empowerment after GC [12]. It is therefore possible that it is this subgroup of patients who are driving the significant association between indication for referral and change in empowerment after GC in the present study.

While specific psychiatric diagnosis had no statistically significant impact on change in levels of empowerment associated with $\mathrm{GC}$, the effect size was moderate $\left(\eta^{2}=\right.$ 0.056), suggesting the possibility that a larger sample size may have yielded a statistically significant association between greater increases in empowerment for those with bipolar disorder, anxiety, or depression as compared with individuals with schizophrenia. Some support for this idea comes from previous research, which demonstrated psychiatric GC reduces internalized stigma with a larger effect size for people with bipolar disorder and schizoaffective disorder as compared with those with schizophrenia [17].

Our finding that baseline (T1) GCOS scores predict the degree of change in empowerment after GC is to our 
Table 4 Demographics and patient characteristics for individuals above and below baseline GCOS threshold predicted to achieve a MCID (GCOS change score of 10.3).

\begin{tabular}{|c|c|c|c|}
\hline & $\begin{array}{l}\mathrm{T} 1 \mathrm{GCOS} \leq 131 \\
N=268\end{array}$ & $\begin{array}{l}\text { T1 GCOS }>131 \\
N=39\end{array}$ & $p$ \\
\hline Age & & & 0.025 \\
\hline $13-40$ & $134(50.0)$ & $27(69.2)$ & \\
\hline $41-77$ & $134(50.0)$ & $12(30.8)$ & \\
\hline Sex & & & 0.891 \\
\hline Male & $43(16.1)$ & 7 (17.9) & \\
\hline Female & $224(83.8)$ & $32(82.1)$ & \\
\hline Ethnicity & & & 0.883 \\
\hline European & $181(70.7)$ & $27(69.2)$ & \\
\hline Asian & $40(15.6)$ & $6(15.4)$ & \\
\hline Mixed & $29(11.3)$ & $5(12.8)$ & \\
\hline African & $3(1.2)$ & $0(0.0)$ & \\
\hline Other & $3(1.2)$ & $1(2.6)$ & \\
\hline Mode of referral & & & 0.217 \\
\hline Self-referral & $103(38.4)$ & $11(28.2)$ & \\
\hline Healthcare provider & $165(61.6)$ & $28(71.8)$ & \\
\hline \multicolumn{4}{|l|}{ Primary indication ${ }^{\mathrm{a}}$} \\
\hline Recurrence risk & $131(48.9)$ & $16(41.0)$ & 0.359 \\
\hline Understanding causes & $166(61.9)$ & $23(59.0)$ & 0.722 \\
\hline Protective factors & $65(24.3)$ & $16(41.0)$ & 0.026 \\
\hline Had genetic testing & $4(1.5)$ & $0(0.0)$ & 1.0 \\
\hline Pregnancy related & $13(4.9)$ & $4(10.3)$ & 0.248 \\
\hline Other & $6(2.2)$ & $0(0.0)$ & 1.0 \\
\hline Unsure & $17(6.3)$ & $3(7.7)$ & 0.728 \\
\hline History of mental illness & & & 0.016 \\
\hline Personal history & $221(82.5)$ & $38(97.4)$ & \\
\hline Family history only & $47(17.5)$ & $1(2.6)$ & \\
\hline \multicolumn{4}{|l|}{ Personal history ${ }^{\mathrm{b}}$} \\
\hline Schizophrenia & $4(4.3)$ & $1(5.6)$ & 0.495 \\
\hline Bipolar disorder & $24(25.5)$ & $6(33.3)$ & 0.243 \\
\hline Schizoaffective & $3(3.2)$ & $1(5.6)$ & 0.421 \\
\hline Anxiety & $14(14.9)$ & $3(16.7)$ & 0.462 \\
\hline Depression & $46(48.9)$ & 7 (38.9) & 0.904 \\
\hline Other & $3(3.2)$ & 0 & 1.0 \\
\hline
\end{tabular}

$P$ values from Pearson chi-square, or Fisher's exact test when appropriate.

andividuals can have more than one primary indication.

${ }^{b}$ Personal history is only for those individuals with one diagnosis. If they have more than one diagnosis they are not included.

knowledge — novel—and raises interesting possibilities for future research and clinical practice, as described below. The finding that those who indicated a desire to discuss protective factors had higher baseline GCOS scores makes a degree of intuitive sense (these individuals are ready to talk about protecting their mental health for the future), but that older individuals and those with only a family history of mental illness (rather than a personal history) had lower baseline GCOS scores may need deeper exploration (e.g., qualitative study) to fully understand.

\section{Study limitations}

The majority of patients were female, European, and had a personal history of mental illness. The psychiatric diagnoses were per patient report, and not confirmed via medical records. Furthermore, GCOS scores were measured $\sim 1$ month after GC, but longer term effects were not assessed.

\section{Practice implications}

Our data demonstrate that patients with a range of different ethnicities, sexes, and diagnoses benefit from psychiatric GC, and provide some initial insight into some of the patient- and session-related variables that could influence GC outcomes. Although additional research (as described below) is required, our data may lay the foundations for considering the clinical use of baseline GCOS scores for the purpose of identifying patients to refer to GC, and/or triaging those already referred (i.e., providing first available appointments to those with the lowest scores).

\section{Future research}

The growing body of data reporting on using the GCOS in different practice settings, opens the opportunity to consider comparing how different specialties and practice models within GC compare in terms of their impact on empowerment and its subdomains. Future work could build on the data we report here regarding baseline GCOS scoresspecifically, studies could explore the possibility of triaging those referred for GC based on this variable. For example, it could be worthwhile to explore the outcomes of prioritizing (e.g., providing first available appointments to) those with lower baseline (T1) GCOS scores, given our data suggesting that these individuals benefit most from GC (as evidenced by greater improvements in GCOS scores after GC). As well, studies exploring the use of tools like the GCOS to identify patients who would not typically be referred/eligible but who could benefit from GC may be warranted (e.g., those with family history of cancer who would not be prioritized for GC services using current risk-based triage models could perhaps be offered appointments if they had a GCOS score below a given threshold - importantly, baseline GCOS scores that correlate with MCID may vary in different contexts). Future research could also usefully explore the wide variety of additional factors not explored here (e.g., coping style, personality characteristics) that may influence patient outcomes. 
Acknowledgements The authors thank Dr Arianne Albert for her assistance regarding the statistical analyses and Caitlin Slomp for her insight throughout the research process. This research was supported by Janelle Villiers, and the Joan H. Marks Graduate Program in Human Genetics at Sarah Lawrence College. JA was supported by the Canada Research Chairs program, and the British Columbia Mental Health and Substance use Services. The authors thank the members of the Translational Psychiatric Genetics Group for their varied support and contributions.

\section{Compliance with ethical standards}

Conflict of interest The authors declare that they have no conflict of interest.

Publisher's note Springer Nature remains neutral with regard to jurisdictional claims in published maps and institutional affiliations.

\section{References}

1. Redlinger-Grosse K, Veach PMC, Cohen S, LeRoy BS, MacFarlane IM, Zierhut H. Defining our clinical practice: the identification of genetic counseling outcomes utilizing the reciprocal engagement model. J Genet Couns. 2016;25:239-57.

2. Zierhut HA, Shannon KM, Cragun DL, Cohen SA. Elucidating genetic counseling outcomes from the perspective of genetic counselors. J Genet Couns. 2016;25:993-1001.

3. Burke W, Culver JO, Bowen D, Lowry D, Durfy S, McTiernan A, et al. Genetic counseling for women with an intermediate family history of breast cancer. Am J Med Genet. 2000;90:361-8.

4. Cabrera E, Blanco I, Yagüe C, Zabalegui A. The impact of genetic counseling on knowledge and emotional responses in Spanish population with family history of breast cancer. Patient Educ Couns. 2010;78:382-8.

5. Cragun D, Camperlengo L, Robinson E, Caldwell M, Kim J, Phelan $\mathrm{C}$, et al. Differences in BRCA counseling and testing practices based on ordering provider type. Genet Med. 2015;17:51-7.

6. Oberguggenberger A, Sztankay M, Morscher RJ, SpernerUnterweger B, Weber I, Hubalek M, et al. Psychosocial outcomes and counselee satisfaction following genetic counseling for hereditary breast and ovarian cancer: a patient-reported outcome study. J Psychosom Res. 2016;89:39-45.

7. Madlensky L, Trepanier AM, Cragun D, Lerner B, Shannon KM, Zierhut $\mathrm{H}$. A rapid systematic review of outcomes studies in genetic counseling. J Genet Couns. 2017;26:361-78.

8. Ison HE, Ware SM, Schwantes-An T-H, Freeze S, Elmore L, Spoonamore KG. The impact of cardiovascular genetic counseling on patient empowerment. J Genet Couns. 2019;28:570-7.

9. Buchanan AH, Datta SK, Skinner CS, Hollowell GP, Beresford HF, Freeland T, et al. Randomized trial of telegenetics vs. inperson cancer genetic counseling: cost, patient satisfaction and attendance. J Genet Couns. 2015;24:961-70.

10. Schwartz MD, Valdimarsdottir HB, Peshkin BN, Mandelblatt J, Nusum R, Huang A-T, et al. Randomized noninferiority trial of telephone versus in-person genetic counseling for hereditary breast and ovarian cancer. J Clin Oncol. 2014;32:618-26.

11. Slomp C, Morris E, Inglis A, Lehman A, Austin J. Patient outcomes of genetic counseling: assessing the impact of different approaches to family history collection. Clin Genet. 2018;93:830-6.

12. Borle K, Morris E, Inglis A, Austin J. Risk communication in genetic counseling: exploring uptake and perception of recurrence numbers, and their impact on patient outcomes. Clin Genet. 2018;94:239-45.
13. Morris E, Best J, Inglis A, Austin J. Impact of the physical environment on patient outcomes of genetic counseling: an exploratory study. J Genet Couns. 2019;28:760-6.

14. Austin JC, Honer WG. Psychiatric genetic counselling for parents of individuals affected with psychotic disorders: a pilot study. Early Intervention Psychiatry. 2008;2:80-89.

15. Costain G, Esplen MJ, Toner B, Scherer SW, Meschino WS, Hodgkinson KA, et al. Evaluating genetic counseling for individuals with schizophrenia in the molecular age. Schizophrenia Bull. 2014;40:78-87.

16. Costain G, Esplen MJ, Toner B, Hodgkinson KA, Bassett AS. Evaluating genetic counseling for family members of individuals with schizophrenia in the molecular age. Schizophrenia Bull. 2014:40:88-99.

17. Hippman C, Ringrose A, Inglis A, Cheek J, Albert A, Remick R, et al. A pilot randomized clinical trial evaluating the impact of genetic counseling for serious mental illnesses. J Clin Psychiatry. 2016;77:190-8.

18. Inglis A, Koehn D, Mcgillivray B, Stewart SE, Austin J. Evaluating a unique, specialist psychiatric genetic counseling clinic: uptake and impact. Clin Genet. 2015;87:218-24.

19. Moldovan R, Pintea S, Austin J. The efficacy of genetic counseling for psychiatric disorders: a meta-analysis. J Genet Couns. 2017;26:1341-7.

20. Costal Tirado A, McDermott AM, Thomas C, Ferrick D, Harris J, Edwards A, et al. Using patient-reported outcome measures for quality improvement in clinical genetics: an exploratory study. J Genet Couns. 2017;26:1017-28.

21. Mcallister M, Wood A, Dunn G, Shiloh S, Todd C. The Genetic Counseling Outcome Scale: a new patient-reported outcome measure for clinical genetics services. Clin Genet. 2011;79:413-24.

22. Resta R, Bowles Biesecker B, Bennett R, Blum S, Hahn SE, Strecker $\mathrm{MN}$, et al. A new definition of genetic counseling: national society of genetic counselors' task force report. J Genet Couns. 2006;15:77-83.

23. Austin, JC. Evidence-based genetic counseling for psychiatric disorders: a road map. Cold Spring Harb Perspect Med. 2019. https://doi.org/10.1101/cshperspect.a036608.

24. Harris PA, Taylor R, Thielke R, Payne J, Gonzalez N, Conde JG. Research electronic data capture (REDCap) - a metadatadriven meth- odology and workflow process for providing translational research informatics support. J Biomed Inform. 2009;42:377-81.

25. Thomas C, Mcallister M. Establishing the minimum clinically important difference for the Genetic Counseling Outcome Scale (GCOS-24). J Genet Couns. 2019;28:1-8.

26. Thomas C, Mcallister M. Establishing the minimum clinically important difference for the Genetic Counseling Outcome Scale (GCOS-24). J Genet Couns. 2019;28:1-8.

27. Christensen KD, Roberts JS, Zikmund-Fisher BJ, Kardia SLR, McBride CM, Linnenbringer E, et al. Associations between selfreferral and health behavior responses to genetic risk information. Genome Med. 2015;7:1-11.

28. Snyder DC, Sloane R, Lobach D, Lipkus IM, Peterson B, Kraus $\mathrm{W}$, et al. Differences in baseline characteristics and outcomes at 1and 2-year follow-up of cancer survivors accrued via self-referral versus cancer registry in the FRESH START Diet and exercise trial. Cancer Epidemiol, Biomark Prev. 2008;17:1288-94.

29. Audrain J, Schwartz MD, Lerman C, Hughes C, Peshkin BN, Biesecker B. Psychological distress in women seeking genetic counseling for breast-ovarian cancer risk: the contributions of personality and appraisal. Ann Behav Med. 1998;19:370-7.

30. Henrikson NB, Harris JN, Bowen DJ. Predictors of self-referral into a cancer genetics registry. Cancer Epidemiol Biomark Prev. 2007;16:1387-92. 